\title{
Indefinite trans-Sasakian manifold with semi-symmetric metric connection
}

\author{
Rajendra Prasad ${ }^{1}$ and Sushil Kumar ${ }^{2}$ \\ Department of Mathematics and Astronomy, Lucknow Univesity \\ E-mail: ${ }^{1}$ rp.manpur@rediffmail.com, ${ }^{2}$ sushilmath20@gmail.com
}

\begin{abstract}
The objective of the present paper is to study of indefinite trans-Sasakian manifold with a semi-symmetric metric connection. We have found the relations between curvature tensors, Ricci curvature tensors and scalar curvature of indefinite trans-Sasakian manifolds with semisymmetric metric connection and with metric connection. Also, we have proved some results on quasi-projectively flat and $\varphi$-projectively flat manifolds with respect to semi-symmetric metric connection. It is shown that the manifold satisfying $\bar{R} \cdot \bar{S}=0$ is an $\eta$-Einstein manifold if $\alpha=0$ and $\beta=$ constant. It is also proved that the manifold satisfying $\bar{P} \cdot \bar{S}=0$ is an $\eta$-Einstein manifold if $\alpha=0$ and $\beta=$ constant. Finally, we have obtained the conditions for the manifold with semi-symmetric metric connection to be conformally flat and $\xi$-conformally flat.
\end{abstract}

2010 Mathematics Subject Classification. 53C25. 53C50

Keywords. Semi-symmetric metric connection, Curvature tensor, Ricci-curvature tensor.

\section{Introduction}

The notion of semi-symmetric linear connection on a differentiable manifold was introduced by Friedman and Schouten in 1924 [8] and metric connection with a torsion on a Riemannain manifold was studied by Hayden in 1932 [9]. Semi-symmetric metric connection on a Riemannain manifold had been given by Yano in 1970 [17] and later studied by K.S.Amur and S.S.Pujar [11], C.S.Bagewadi [1], U. C. De et al [7], Sharafuddin and Hussain [10] and others. U.C.De [7] and C.S.Bagewadi et al. $[2,3,14]$ previously provided some results on the conservativeness of Projective, Pseudo projective, Conformal, Concircular, Quasi conformal curvature tensors on K-contact, Kenmotsu and transSasakian manifolds.

A new class of $n$-dimensional almost contact manifold namely trans-Sasakian manifold was introduced by J. A. Oubina in 1985 [15] and further study about the local structures of transSasakian manifolds was carried by J.C.Marrero [13]. As a natural generalization of both Sasakian and Kenmotsu manifolds, the notion of trans-Sasakian manifolds, which are closely related to the locally conformal Kahler manifolds introduced by Oubina [15 ]. Trans-Sasskian manifold of type $(0,0),(\alpha, 0)$, and $(0, \beta)$ are, respectively, called the cosymplectic, $\alpha$-Sasakian, and $\beta$-Kenmotsu manifolds, with $\alpha, \beta$ being scalar functions. In particular, if $\alpha=0, \beta=1 ; \alpha=1, \beta=0$; then a trans-Sasakian manifold becomes Kenmotsu and Sasakian manifolds respectively. Hence trans -Saskian structures give a large class of generalized Quasi- Sasakian structures. The concept of indefinite Sasakian manifolds was introduced by A. Bejancu and K. L. Duggal [4] and further study was taken up by Xufend and Xiaoli[18], Rakesh Kumar et al. [12]. De and Sarkar [6].

In this present paper we shall introduce relation between metric connection and semi-symmetric metric connection in an $n$-dimensional indefinite trans Sasakian manifold $M^{n}$. Also, we have 
proved some results on curvature tensors, Ricci curvature tensors, scalar curvatures, quasi projectively flat, $\varphi$-projectively flat, $\bar{R} \cdot \bar{S}=0, \bar{P} \cdot \bar{S}=0$, Weyl conformally flat, Weyl $\xi$-conformally flat respectively in $n$-dimensional indefinite trans-Sasakian manifold $M^{n}$ with a semi-symmetric metric connection.

\section{Preliminaries}

An $n$-dimensional smooth manifold $\left(M^{n}, g\right)$ is said to be an indefinite almost contact metric manifold [5], if it admits a $(1,1)$ tensor field $\varphi$, a structure vector field $\xi$, a 1 -form $\eta$ and an indefinite metric $g$ such that

$$
\begin{gathered}
\varphi^{2} X=-X+\eta(X) \xi, \varphi \xi=0, \eta(\varphi X)=0, \\
\eta(\xi)=1, \\
g(\xi, \xi)=\varepsilon \\
\eta(X)=\varepsilon g(X, \xi), \\
g(\varphi X, \varphi Y)=g(X, Y)-\varepsilon \eta(X) \eta(Y),
\end{gathered}
$$

for all vector fields $X, Y$ on $M^{n}$, where $\varepsilon$ is 1 or -1 according as $\xi$ is space like or time like vector field and rank $\varphi$ is $n-1$. If

$$
d \eta(X, Y)=g(X, \varphi Y)
$$

then $M^{n}(\varphi, \xi, \eta, g)$ is called an indefinite contact metric manifold. An indefinite almost contact metric manifold is called an indefinite trans-Sasakian manifold if

$$
\left(\nabla_{X} \varphi\right) Y=\alpha\{g(X, Y) \xi-\varepsilon \eta(Y) X\}+\beta\{g(\varphi X, Y) \xi-\varepsilon \eta(Y) \varphi X\}
$$

for any $X, Y \in \Gamma\left(T M^{n}\right)$, where $\nabla$ is metric connection of indefinite metric $g, \alpha$ and $\beta$ are smooth functions on $M^{n}$.

From equations $(2.1),(2.2),(2.3),(2.4),(2.5)$ and $(2.7)$, we have

$$
\begin{gathered}
\nabla_{X} \xi=\varepsilon\{-\alpha \varphi X+\beta(X-\eta(X) \xi)\} \\
\left(\nabla_{X} \eta\right) Y=-\alpha g(\varphi X, Y)+\beta\{g(X, Y)-\varepsilon \eta(X) \eta(Y)\} .
\end{gathered}
$$

Moreover, on such a indefinite trans-Sasakian manifold $M^{n}$ of dimension $n$ with structure $M^{n}(\varphi, \xi, \eta, g)$ the following relations holds:

$$
\begin{aligned}
R(X, Y) \xi= & \left(\alpha^{2}-\beta^{2}\right)\{\eta(Y) X-\eta(X) Y\} \\
& +2 \alpha \beta\{\eta(Y) \varphi X-\eta(X) \varphi Y\}+\varepsilon\{(Y \alpha) \varphi X \\
& \left.-(X \alpha) \varphi Y+(Y \beta) \varphi^{2} X-(X \beta) \varphi^{2} Y\right\}, \\
R(\xi, Y) Z=\quad & \\
& \left.\alpha^{2}-\beta^{2}\right)\{\varepsilon g(Y, Z) \xi-\eta(Z) Y\}+2 \alpha \beta\{\varepsilon g(Y, \varphi Z) \xi \\
+ & \eta(Z) \varphi Y\}+\varepsilon(Z \alpha) \varphi Y+\varepsilon g(Y, \varphi Z)(\operatorname{grad} \alpha) \\
- & \varepsilon g(\varphi Y, \varphi Z)(\operatorname{grad} \beta)+\varepsilon(Z \beta)\{Y-\eta(Z) \xi\},
\end{aligned}
$$




$$
\begin{gathered}
S(Z, \xi)=\left\{(n-1)\left(\alpha^{2}-\beta^{2}\right)-\varepsilon(\xi \beta)\right\} \eta(Z)-\varepsilon(\varphi Z) \alpha-\varepsilon(n-2)(Z \beta), \\
S(\xi, \xi)=(n-1)\left(\alpha^{2}-\beta^{2}\right)-\varepsilon(n-1)(\xi \beta), \\
Q \xi=\varepsilon(n-1)\left(\alpha^{2}-\beta^{2}\right) \xi-(\xi \beta) \xi+\varepsilon \varphi(g r a d \alpha)-\varepsilon(n-2)(g r a d \beta), \\
C(X, Y) Z=\quad R(X, Y) Z-\frac{1}{(n-2)}[S(Y, Z) X-S(X, Z) Y \\
+g(Y, Z) Q X-g(X, Z) Q Y]+\frac{r}{(n-1)(n-2)} \\
{[g(Y, Z) X-g(X, Z) Y],} \\
g(R(\xi, Y) Z, \xi)=\left[\left(\alpha^{2}-\beta^{2}\right)-\varepsilon(\xi \beta)\right] g(Y, Z) \\
+\left[(\xi \beta)-\varepsilon\left(\alpha^{2}-\beta^{2}\right)\right] \eta(Y) \eta(Z) \\
-[2 \alpha \beta+\varepsilon(\xi \alpha)] g(\varphi Y, Z), \\
r=S\left(e_{i}, e_{i}\right)=\sum_{i=1}^{n} \varepsilon_{i}{ }^{\prime} R\left(e_{i}, e_{i}, e_{i}, e_{i}\right) .
\end{gathered}
$$

\section{Semi-symmetric metric connection}

A affine connection $\bar{\nabla}$ in $M^{n}$ is called semi-symmetric connection [10], if its torsion tensor

$$
\bar{T}(X, Y)=\bar{\nabla}_{X} Y-\bar{\nabla}_{Y} X-[X, Y],
$$

satisfies

$$
\bar{T}(X, Y)=\eta(X) Y-\eta(Y) X .
$$

Moreover, a semi-symmetric connection is called semi-symmetric metric connection if

$$
\left(\bar{\nabla}_{X} g\right)(Y, Z)=0 .
$$

If $\nabla$ is metric connection and $\bar{\nabla}$ is the semi-symmetric metric with non-vanishing torsion tensor $T$ in $M^{n}$, then we have

$$
\begin{gathered}
T(X, Y)=\eta(Y) X-\eta(X) Y \\
\bar{\nabla}_{X} Y-\nabla_{X} Y=\frac{1}{2}\left[T(X, Y)+T^{\mid}(X, Y)+T^{\mid}(X, Y)\right],
\end{gathered}
$$

where

$$
g(T(Z, X), Y)=g\left(T^{\mid}(X, Y), Z\right) .
$$

By using equations (2.4), (3.4) and (3.6), we get

$$
\begin{gathered}
g\left(T^{\mid}(X, Y), Z\right)=g(\eta(X) Z-\eta(Z) X, Y), \\
g\left(T^{\mid}(X, Y), Z\right)=\eta(X) g(Z, Y)-\varepsilon g(X, Y) g(\xi, Z), \\
T^{\mid}(X, Y)=\eta(X) Y-\varepsilon g(X, Y) \xi,
\end{gathered}
$$




$$
T^{\mid}(Y, X)=\eta(Y) X-\varepsilon g(X, Y) \xi,
$$

From equations (3.4), (3.5), (3.7) and (3.8), we get

$$
\bar{\nabla}_{X} Y=\nabla_{X} Y+\eta(Y) X-\varepsilon g(X, Y) \xi .
$$

Let $M^{n}$ be an $n$-dimensional indefinite trans-Sasakian manifold and $\nabla$ be the metric connection on $M^{n}$. The relation between the semi-symmetric metric connection $\bar{\nabla}$ and the metric connection $\nabla$ on $M^{n}$ is given by

$$
\bar{\nabla}_{X} Y=\nabla_{X} Y+\eta(Y) X-\varepsilon g(X, Y) \xi .
$$

\section{Curvature tensor on indefinite trans-Sasakian manifold with semi-symmetric metric connection}

Let $M^{n}$ be an $n$-dimensional indefinite trans-Sasakian manifold. The curvature tensor $\bar{R}$ of $M^{n}$ with respect to the semi-symmetric metric connection $\bar{\nabla}$ is defined by

$$
\bar{R}(X, Y) Z=\bar{\nabla}_{X} \bar{\nabla}_{Y} Z-\bar{\nabla}_{Y} \bar{\nabla}_{X} Z-\nabla_{[X, Y]}^{-} Z .
$$

By using equations (2.2), (2.4), (3.9) and (4.1), we get

$$
\begin{aligned}
\bar{R}(X, Y) Z= & R(X, Y) Z+(2 \beta+\varepsilon)[g(X, Z) Y-g(Y, Z) X] \\
& +(\beta+\varepsilon)[\eta(X) g(Y, Z)-\eta(Y) g(X, Z)] \xi \\
& +(1+\beta \varepsilon) \eta(Z)[\eta(Y) X-\eta(X) Y] \\
& -\alpha[g(\varphi X, Z) Y-g(\varphi Y, Z) X-g(Y, Z) \varphi X+g(X, Z) \varphi Y],
\end{aligned}
$$

where

$$
R(X, Y) Z=\nabla_{X} \nabla_{Y} Z-\nabla_{Y} \nabla_{X} Z-\nabla_{[X, Y]} Z,
$$

is the Riemannian curvature tensor of metric connection $\nabla$.

Taking inner product of equation (4.2) with $U$ and using equation (2.4), we have

$$
\begin{aligned}
& ' \\
= & ' R(X, Y, Z, U) \\
& +(1+\varepsilon \beta)[\eta(X) g(Y, Z)-\eta(Y) g(X, Z)] \eta(U) \\
& +(1+\varepsilon \beta)[\eta(Y) g(X, U)-\eta(X) g(Y, U,)] \eta(Z) \\
& -\alpha[g(\varphi X, Z) g(Y, U)-g(\varphi Y, Z) g(X, U)-g(Y, Z) g(\varphi X, U) \\
& +g(X, Z) g(\varphi Y, U)],
\end{aligned}
$$

where ' $\bar{R}(X, Y, Z, U)=g(\bar{R}(X, Y) Z, U)$ and ' $R(X, Y, Z, U)=g(R(X, Y) Z, U)$.

Let $\left\{e_{1}, e_{2}, \ldots \ldots \ldots \ldots . . ., e_{n-1}, \xi\right\}$ be a local orthonormal basis of vector fields on indefinite transSasakian manifold $M^{n}$, then $\left\{\varphi e_{1}, \varphi e_{2}, \ldots \ldots \ldots \ldots, \varphi e_{n-1}, \xi\right\}$ is a also local orthonormal basis of vector 
fields on indefinite trans-Sasakian manifold $M^{n}$. Now, putting $X=U=e_{\mathrm{i}}$ in the equation (4.3) and taking summation over $i, 1 \leq i \leq n-1$, we get

$$
\begin{aligned}
& \sum_{i=1}^{n-1} ' \bar{R}\left(e_{i}, Y, Z, e_{i}\right) \\
= & \sum_{i=1}^{n-1} ' R\left(e_{i}, Y, Z, e_{i}\right)+(2 \beta+\varepsilon) \sum_{i=1}^{n-1}\left[g\left(e_{i}, Z\right) g\left(Y, e_{i}\right)-g(Y, Z) g\left(e_{i}, e_{i}\right)\right] \\
& +(\beta+\varepsilon) \sum_{i=1}^{n-1}\left[\varepsilon g(Y, Z) g\left(e_{i}, \xi\right) g\left(\xi, e_{i}\right)-\eta(Y) g\left(e_{i}, Z\right) g\left(\xi, e_{i}\right)\right] \\
& +(\beta \varepsilon+1) \sum_{i=1}^{n-1}\left[\eta(Z) \eta(Y) g\left(e_{i}, e_{i}\right)-\varepsilon \eta(Z) g\left(e_{i}, \xi\right) g\left(Y, e_{i}\right)\right] \\
& -\alpha \sum_{i=1}^{n-1}\left[g\left(e_{i} \varphi, Z\right) g\left(Y, e_{i}\right)-g(\varphi Y, Z) g\left(e_{i}, e_{i}\right)-g(Y, Z) g\left(\varphi e_{i}, e_{i}\right)\right. \\
& \left.+g\left(e_{i}, Z\right) g\left(\varphi Y, e_{i}\right)\right] .
\end{aligned}
$$

We have using

$$
\begin{gathered}
S(Y, Z)=\sum_{i=1}^{n} \varepsilon_{i}{ }^{\prime} R\left(e_{i}, Y, Z, e_{i}\right), g(Y, Z)=\sum_{i=1}^{n} \varepsilon_{i} g\left(Y, e_{i}\right) g\left(e_{i}, Z\right), \\
\bar{S}(Y, Z)=\sum_{i=1}^{n} \varepsilon_{i}{ }^{\prime} \bar{R}\left(e_{i}, Y, Z, e_{i}\right) .
\end{gathered}
$$

Also

$$
\begin{gathered}
\sum_{i=1}^{n-1} ' \bar{R}\left(e_{i}, Y, Z, e_{i}\right)=\bar{S}(Y, Z)-\varepsilon \prime \bar{R}(\xi, Y, Z, \xi), \\
\sum_{i=1}^{n-1} ' R\left(e_{i}, Y, Z, e_{i}\right)=S(Y, Z)-\varepsilon \prime R(\xi, Y, Z, \xi), \\
\sum_{i=1}^{n-1} g\left(e_{i}, Z\right) g\left(Y, e_{i}\right)=g(Y, Z)-\varepsilon g(\xi, Z) g(Y, \xi), \\
\sum_{i=1}^{n-1} g\left(e_{i}, e_{i}\right)=n-1, \\
\sum_{i=1}^{n-1} g\left(e_{i}, \xi\right) g\left(\xi, e_{i}\right)=0 \\
\sum_{i=1}^{n-1} g\left(e_{i}, Z\right) g\left(\xi, e_{i}\right)=0
\end{gathered}
$$




$$
\begin{gathered}
\sum_{i=1}^{n-1} g\left(\varphi e_{i}, Z\right) g\left(Y, e_{i}\right)=g(\varphi Y, Z), \\
\sum_{i=1}^{n-1} g\left(\varphi e_{i}, e_{i}\right)=0, \\
\sum_{i=1}^{n-1} g\left(\varphi e_{i}, \varphi e_{i}\right)=n-1, \\
\sum_{i=1}^{n-1} g\left(\varphi e_{i}, Z\right) g\left(Y, \varphi e_{i}\right)=g(Y, Z)-\varepsilon g(\xi, Z) g(Y, \xi) .
\end{gathered}
$$

Hence, by virtue of equations (4.4) and (4.7) - (4.16), we get

$$
\begin{aligned}
\bar{S}(Y, Z)= & S(Y, Z)-[(2 \beta+\varepsilon)(n-2)+\beta] g(Y, Z) \\
& +(1+\varepsilon \beta)(n-2) \eta(Z) \eta(Y)+\alpha(n-2) g(\varphi Y, Z),
\end{aligned}
$$

where $\bar{S}$ and $S$ are the Ricci tensor of connection $\bar{\nabla}$ and $\nabla$, respectively in $M^{n}$.

$$
\bar{Q} Y=Q Y-[(2 \beta+\varepsilon)(n-2)+\beta] Y+(\varepsilon+\beta)(n-2) \eta(Y) \xi+\alpha(n-2) \varphi Y,
$$

where $\bar{Q}$ and $Q$ are Ricci operator with respect to the semi-symmetric metric connection and metric connection respectively and are define as $g(\bar{Q} Y, Z)=\bar{S}(Y, Z)$ and $g(Q Y, Z)=S(Y, Z)$ respectively.

Replace $Y=\xi$ in (4.18) and using (2.14), we get

$$
\begin{aligned}
\bar{Q} \xi= & \varepsilon(n-1)\left(\alpha^{2}-\beta^{2}\right) \xi-(\xi \beta) \xi+\varepsilon \varphi(\operatorname{grad} \alpha) \\
& -\varepsilon(n-2)(\operatorname{grad} \beta)-\beta(n-1) \xi .
\end{aligned}
$$

Putting $Y=Z=e_{i}$ and taking summation over $i, 1 \leq i \leq n-1$ in equation (4.17), using equations (2.13), (2.17), (4.10), (4.11) and (4.14), we get

$$
\bar{r}=r-(n-1)[(2 \beta+\varepsilon)(n-2)+2 \beta],
$$

where $\bar{r}$ and $r$ are the scalar curvatures of the connection $\bar{\nabla}$ and $\nabla$, respectively.

Replace $Y \rightarrow \varphi Y$ in equation (4.17), we get

$$
\begin{aligned}
\bar{S}(\varphi Y, Z)= & S(\varphi Y, Z)-[(2 \beta+\varepsilon)(n-2)+\beta] g(\varphi Y, Z) \\
& -\alpha(n-2) g(Y, Z)+\varepsilon \alpha(n-2) \eta(Z) \eta(Y) .
\end{aligned}
$$

Using equations (2.1), (2.2), (2.4) and (2.12) in equation (4.21), we get

$$
\bar{S}(\varphi Y, \xi)=S(\varphi Y, \xi)=-\varepsilon\left(\varphi^{2} Y\right) \alpha-\varepsilon(n-2)(\varphi Y) \beta .
$$

Using equations (2.1), (2.2), (2.4), (2.12), (2.13) and (4.17), we get

$$
\begin{aligned}
\bar{S}(Y, \xi)= & {\left[(n-1)\left(\alpha^{2}-\beta^{2}\right)-\varepsilon(\xi \beta)-\varepsilon \beta(n-1)\right] \eta(Y) } \\
& -\varepsilon(\varphi Y) \alpha-\varepsilon(n-2)(Y \beta),
\end{aligned}
$$




$$
\begin{aligned}
& \bar{S}(\xi, \xi)=(n-1)\left(\alpha^{2}-\beta^{2}\right)-\varepsilon \beta(n-1)-\varepsilon(n-1)(\xi \beta), \\
& \bar{S}(\operatorname{grad} \alpha, \xi)=\varepsilon(n-1)\left(\alpha^{2}-\beta^{2}\right)(\xi \alpha)-\beta(n-1)(\xi \alpha)-(\xi \alpha)(\xi \beta) \\
& -\varepsilon(\varphi \operatorname{grad} \alpha) \alpha-\varepsilon(n-2) \operatorname{g}(\operatorname{grad} \alpha, \operatorname{grad} \beta) \text {, } \\
& \bar{S}(\operatorname{grad} \beta, \xi)=\varepsilon(n-1)\left(\alpha^{2}-\beta^{2}\right)(\xi \beta)-\beta(n-1)(\xi \beta)-(\xi \beta)^{2} \\
& -\varepsilon(\varphi \operatorname{grad} \beta) \alpha-\varepsilon(n-2)(\operatorname{grad} \beta)^{2} .
\end{aligned}
$$

From equation (4.2) by the cyclic permutations of $X, Y$ and $Z$, we get

$$
\begin{aligned}
& R(Y, Z) X \\
= & R(Y, Z) X+(\varepsilon+2 \beta)[g(Y, X) Z-g(Z, X) Y] \\
& +(\varepsilon+\beta)[\eta(Y) g(Z, X)-\eta(Z) g(Y, X)] \xi \\
& +(1+\beta \varepsilon) \eta(X)[\eta(Z) Y-\eta(Y) Z] \\
& -\alpha[g(\varphi Y, X) Z-g(\varphi Z, X) Y-g(Z, X) \varphi Y+g(Y, X) \varphi Z], \\
& -R(Z, X) Y \\
= & R(Z, X) Y+(2 \beta+\varepsilon)[g(Z, Y) X-g(X, Y) Z] \\
& +(\varepsilon+\beta)[\eta(Y)[\eta(Z) g(X, Y)-\eta(X) g(Y, Z)] \xi \\
& +(1+\varepsilon \beta) \eta(Y)[\eta(X) Z-\eta(Z) X] \\
& -\alpha[g(\varphi Z, Y) X-g(\varphi X, Y) Z-g(X, Y) \varphi Z+g(Z, Y) \varphi X] .
\end{aligned}
$$

Adding equations (4.2), (4.27) and (4.28), using the Bianchi' first identity, we get

$$
\begin{aligned}
& \bar{R}(X, Y) Z+\bar{R}(Y, Z) X+\bar{R}(Z, X) Y \\
= & 2 \alpha[g(\varphi Y, Z) X+g(\varphi Z, X) Y+g(\varphi X, Y) Z] .
\end{aligned}
$$

If $\alpha=0$ in equation (4.29), we have

$$
\bar{R}(X, Y) Z+\bar{R}(Y, Z) X+\bar{R}(Z, X) Y=0 .
$$

Theorem 4.1. Let $M^{n}$ be an $n$-dimensional indefinite trans-Sasakian manifold with semi-symmetric metric connection $\bar{\nabla}$ and curvature tensor $\bar{R}$ satisfies Bianchi first identity if and only if $\alpha=0$, i.e. $M^{n}$ is a $\beta$-Kenmotsu manifold.

Now, from equation (4.3) interchanging $X$ and $Y$, we get

$$
\begin{aligned}
& ' \bar{R}(Y, X, Z, U) \\
= & ' R(Y, X, Z, U)+(\varepsilon+2 \beta)[g(Y, Z) g(X, U)-g(X, Z) g(Y, U)] \\
& +(1+\varepsilon \beta)[\eta(Y) g(X, Z)-\eta(X) g(Y, Z)] \eta(U) \\
& +(1+\varepsilon \beta)[\eta(X) g(Y, U)-\eta(Y) g(X, U,)] \eta(Z) \\
& -\alpha[g(\varphi Y, Z) g(X, U)-g(\varphi X, Z) g(Y, U)-g(X, Z) g(\varphi Y, U) \\
& +g(Y, Z) g(\varphi X, U)] .
\end{aligned}
$$


From equations (4.3) and (4.30), we get

$$
{ }^{\prime} \bar{R}(X, Y, Z, U)=-^{\prime} \bar{R}(Y, X, Z, U),
$$

where

$$
{ }^{\prime} R(X, Y, Z, U)=-{ }^{\prime} R(Y, X, Z, U) .
$$

Again from equation (4.3) interchanging $Z$ and $U$, we get

$$
\begin{aligned}
& ' \bar{R}(X, Y, U, Z) \\
= & ' R(X, Y, U, Z)+(2 \beta+\varepsilon)[g(X, U) g(Y, Z)-g(Y, U) g(X, Z)] \\
& +(1+\varepsilon \beta)[\eta(X) g(Y, U)-\eta(Y) g(X, U)] \eta(Z) \\
& +(1+\varepsilon \beta)[\eta(Y) g(X, Z)-\eta(X) g(Y, Z)] \eta(U) \\
& -\alpha[g(\varphi X, U) g(Y, Z)-g(\varphi Y, U) g(X, Z)-g(Y, U) g(\varphi X, Z) \\
& +g(X, U) g(\varphi Y, Z)] .
\end{aligned}
$$

From equations (4.3) and (4.32), we get

$$
\text { ' } \bar{R}(X, Y, Z, U)=-^{\prime} \bar{R}(X, Y, U, Z),
$$

where

$$
{ }^{\prime} R(X, Y, Z, U)=-^{\prime} R(X, Y, U, Z) .
$$

Again from equation (4.3) interchanging pair of slots, we get

$$
\begin{aligned}
& ' \bar{R}(Z, U, X, Y) \\
= & ' R(Z, U, X, Y)+(2 \beta+\varepsilon)[g(Z, X) g(U, Y)-g(U, X) g(Z, Y)] \\
& +(1+\varepsilon \beta)[\eta(Z) g(U, X)-\eta(U) g(Z, X)] \eta(Y) \\
& +(1+\varepsilon \beta)[\eta(U) g(Z, Y)-\eta(Z) g(U, Y)] \eta(X) \\
& -\alpha[g(\varphi Z, X) g(U, Y)-g(\varphi U, X) g(Z, Y)-g(\varphi Z, Y) g(U, X) \\
& +g(\varphi U, Y) g(Z, X)] .
\end{aligned}
$$

From equations (4.3) and (4.34), we get

$$
\begin{aligned}
& ' \bar{R}(X, Y, Z, U) \\
= & \quad \bar{R}(Z, U, X, Y)+2 \alpha[g(\varphi Z, X) g(U, Y) \\
& +g(\varphi Y, Z) g(X, U)+g(\varphi X, U) g(Y, Z) \\
& +g(\varphi U, Y) g(Z, X)] .
\end{aligned}
$$

If $\alpha=0$ in equation (4.35), we have

$$
{ }^{\prime} \bar{R}(X, Y, Z, U){ }^{\prime} \bar{R}(Z, U, X, Y)
$$

Theorem 4.2. The curvature tensor $\bar{R}$ of type $(0,4)$ of semi-symmetric metric connection $\bar{\nabla}$ is an indefinite trans-Sasakian manifolds is 
(i) Skew symmetric in first two slots.

(ii) Skew symmetric in last two slots.

(iii) Symmetric in pair of slots if and only if $\alpha=0$ in equation (4.35), then $M^{n}$ is a $\beta$-Kenmotsu manifold.

Now, let $\bar{R}(X, Y) Z=0$ in equation (4.2), we get

$$
\begin{aligned}
R(X, Y) Z= & (\varepsilon+2 \beta)[g(Y, Z) X-g(X, Z) Y] \\
& +(\varepsilon+\beta)[\eta(Y) g(X, Z)-\eta(X) g(Y, Z)] \xi \\
& +(1+\varepsilon \beta) \eta(Z)[\eta(X) Y-\eta(Y) X] \\
& +\alpha[g(\varphi X, Z) Y-g(\varphi Y, Z) X-g(Y, Z) \varphi X \\
& +g(X, Z) \varphi Y],
\end{aligned}
$$

Taking the inner product of equation (4.36) with $\xi$ and using (2.4), we get

$$
\begin{aligned}
\varepsilon \eta(R(X, Y) Z)= & \varepsilon \beta[g(Y, Z) \eta(X)-g(X, Z) \eta(Y)] \\
& +\varepsilon \alpha[g(\varphi X, Z) \eta(Y)-g(\varphi Y, Z) \eta(X)] .
\end{aligned}
$$

Using equation (2.4) in equation (4.37), we get

$$
R(X, Y) Z=\beta[g(Y, Z) X-g(X, Z) Y]+\alpha[g(\varphi X, Z) Y-g(\varphi Y, Z) X] .
$$

Theorem 4.3. If the curvature tensor $\bar{R}$ of a semi-symmetric metric connection in an indefinite trans-Sasakian manifold $M^{n}$ vanishes, then the indefinite trans sasakian manifold is of constant curvature if $\alpha=0$. i.e. $M^{n}$ is $\beta$-Kenmotsu manifold.

Now, in equation (4.2) putting $Z=\xi$, using equations (2.1), (2.2), (2.4) and (2.10), we get

$$
\begin{aligned}
\bar{R}(X, Y) \xi= & \left(\alpha^{2}-\beta^{2}-\varepsilon \beta\right)[\eta(Y) X-\eta(X) Y] \\
& +(2 \alpha \beta+\varepsilon \alpha)[\eta(Y) \varphi X-\eta(X) \varphi Y] \\
& +\varepsilon\left[(Y \alpha) \varphi X-(X \alpha) \varphi Y+(Y \beta) \varphi^{2} X-(X \beta) \varphi^{2} Y\right] .
\end{aligned}
$$

Replace $Y=\xi$ in equation (4.39), using equations (2.1), (2.2) and (2.4), we get

$$
\begin{aligned}
\bar{R}(X, \xi) \xi= & \left(\alpha^{2}-\beta^{2}-\varepsilon \beta\right)[X-\eta(X) \xi] \\
& \left.+[2 \alpha \beta+\varepsilon \alpha+\varepsilon(\xi \alpha)] \varphi X+\varepsilon(\xi \beta) \varphi^{2} X\right] .
\end{aligned}
$$

Now, again replace $X=\xi$ in equation (4.39), using equations (2.1), (2.2) and (2.4), we get

$$
\begin{aligned}
\bar{R}(\xi, Y) \xi= & \left(\alpha^{2}-\beta^{2}-\varepsilon \beta\right)[\eta(Y) \xi-Y]-[2 \alpha \beta+\varepsilon \alpha \\
& +\varepsilon(\xi \alpha)] \varphi Y-\varepsilon(\xi \beta) \varphi^{2} Y
\end{aligned}
$$

Replace $Y=X$ in equation (4.41), we get

$$
\begin{aligned}
\bar{R}(\xi, X) \xi= & -\left(\alpha^{2}-\beta^{2}-\varepsilon \beta\right)[X-\eta(X) \xi]-[2 \alpha \beta+\varepsilon \alpha \\
& -\varepsilon(\xi \alpha)] \varphi X-\varepsilon(\xi \beta) \varphi^{2} X .
\end{aligned}
$$

From equations (4.40) and (4.42), we get

$$
\bar{R}(X, \xi) \xi=-\bar{R}(\xi, X) \xi .
$$


Theorem 4.4. Let $M^{n}$ be an $n$-dimensional indefinite trans-Sasakian manifold with a semisymmetric metric connection, then

$$
\begin{aligned}
\bar{R}(X, Y) \xi= & \left(\alpha^{2}-\beta^{2}-\varepsilon \beta\right)[\eta(Y) X-\eta(X) Y] \\
& +(2 \alpha \beta+\varepsilon \alpha)[\eta(Y) \varphi X-\eta(X) \varphi Y] \\
& +\varepsilon\left[(Y \alpha) \varphi X-(X \alpha) \varphi Y+(Y \beta) \varphi^{2} X-(X \beta) \varphi^{2} Y\right] .
\end{aligned}
$$

Lemma 4.5. Let $M^{n}$ be an $n$-dimensional indefinite trans-Sasakian manifold with a semi-symmetric metric connection, then

$$
\begin{gathered}
\left(\bar{\nabla}_{X} \eta\right)(Y)=(\beta+\varepsilon) g(X, Y)-(1+\varepsilon \beta) \eta(X) \eta(Y)-\alpha g(\varphi X, Y), \\
\bar{\nabla}_{X} \xi=(1+\varepsilon \beta) X-(1+\varepsilon \beta) \eta(X) \xi-\varepsilon \alpha(\varphi X) .
\end{gathered}
$$

Proof. By the covariant differentiation of $\eta(Y)$ with respect $X$, we have

$$
\begin{aligned}
\bar{\nabla}_{X} \eta(Y) & =\left(\bar{\nabla}_{X} \eta\right)(Y)+\eta\left(\bar{\nabla}_{X} Y\right), \\
\left(\bar{\nabla}_{X} \eta\right)(Y) & =\bar{\nabla}_{X} \eta(Y)-\eta\left(\bar{\nabla}_{X} Y\right) .
\end{aligned}
$$

By using equations (2.4) and (3.3), we get

$$
\left(\bar{\nabla}_{X} \eta\right)(Y)=\varepsilon g\left(Y, \bar{\nabla}_{X} \xi\right) .
$$

On putting $Y=\xi$ in equation (3.9), we have

$$
\bar{\nabla}_{X} \xi=\nabla_{X} \xi+\eta(\xi) X-\varepsilon g(X, \xi) \xi .
$$

By using equation (2.4), we get

$$
\bar{\nabla}_{X} \xi=\nabla_{X} \xi+X-\eta(X) \xi
$$

From equation (4.47), using equations (2.4), (2.8) and (4.48), we get

$$
\left(\bar{\nabla}_{X} \eta\right)(Y)=(\beta+\varepsilon) g(X, Y)-(1+\varepsilon \beta) \eta(X) \eta(Y)-\alpha g(\varphi X, Y) .
$$

From equations (2.8) and (4.6), we get

$$
\bar{\nabla}_{X} \xi=(1+\varepsilon \beta) X-(1+\varepsilon \beta) \eta(X) \xi-\varepsilon \alpha(\varphi X) .
$$




\section{Quasi-projectively flat indefinite trans-Sasakian manifold with respect to semi-symmetric metric connection}

Let $M^{n}$ be an $n$-dimensional indefinite trans-Sasakian manifold. If there exists a one to one correspondence between each co-ordinate neighbourhood of $M^{n}$ and a domain in Euclidean space such that any geodesic of the indefinite trans-Sasakian manifold corresponds to a straight line in the Euclidean space, then $M^{n}$ is said to be locally projectively flat. The projective curvature tensor $\bar{P}$ with respect to semi-symmetric metric connection is defined by

$$
\bar{P}(X, Y) Z=\bar{R}(X, Y) Z-\frac{1}{(n-1)}[\bar{S}(Y, Z) X-\bar{S}(X, Z) Y] .
$$

Definition 5.1. An indefinite trans-Sasakian manifold $M^{n}$ is said to be quasi-projectively flat with respect to semi-symmetric metric connection, if

$$
g(\bar{P}(\varphi X, Y) Z, \varphi U)=0
$$

where $\bar{P}$ is the projective curvature tensor with respect to semi-symmetric metric connection. From equation (5.1) taking inner product with $U$, we get

$$
\begin{aligned}
g(\bar{P}(X, Y) Z, U)= & g(\bar{R}(X, Y) Z, U)-\frac{1}{(n-1)} \\
& {[\bar{S}(Y, Z) g(X, U)-\bar{S}(X, Z) g(Y, U)] . }
\end{aligned}
$$

Replace $X=\varphi X$ and $U=\varphi U$ in equation (5.3), we get

$$
\begin{aligned}
g(\bar{P}(\varphi X, Y) Z, \varphi U)= & g(\bar{R}(\varphi X, Y) Z, \varphi U)-\frac{1}{(n-1)} \\
& {[\bar{S}(Y, Z) g(\varphi X, \varphi U)-\bar{S}(\varphi X, Z) g(Y, \varphi U)] . }
\end{aligned}
$$

From equations (5.2) and (5.4), we have

$$
g(\bar{R}(\varphi X, Y) Z, \varphi U)=\frac{1}{(n-1)}[\bar{S}(Y, Z) g(\varphi X, \varphi U)-\bar{S}(\varphi X, Z) g(Y, \varphi U)] .
$$

Now, using equations $(2,1),(2.4),(4.17)$ and (4.21) in equation (5.5), we have

$$
\begin{aligned}
& g(R(\varphi X, Y) Z, \varphi U) \\
= & \frac{1}{(n-1)}[S(Y, Z) g(\varphi X, \varphi U)-S(\varphi X, Z) g(Y, \varphi U)] \\
& -\frac{(\beta+\varepsilon)}{(n-1)} g(\varphi X, Z) g(Y, \varphi U)+\frac{(\beta+\varepsilon)}{(n-1)} g(Y, Z) g(\varphi X, \varphi U) \\
& -\frac{(1+\varepsilon \beta)}{(n-1)} \eta(Y) \eta(Z) g(\varphi X, \varphi U)+\frac{\varepsilon \alpha}{(n-1)} \eta(X) \eta(Z) g(Y, \varphi U) \\
- & \frac{\alpha}{(n-1)} g(X, Z) g(Y, \varphi U)-\frac{\alpha}{(n-1)} g(\varphi Y, Z) g(\varphi X, \varphi U) \\
+ & \alpha g(Y, Z) g(X, \varphi U)+\alpha g(\varphi X, Z) g(\varphi Y, \varphi U) .
\end{aligned}
$$


Let $\left\{e_{1}, e_{2}, \ldots \ldots \ldots \ldots, e_{n-1}, \xi\right\}$ be a local orthonormal basis of vector fields on indefinite trans-Sasakian manifold $M^{n}$, then $\left\{\varphi e_{1}, \varphi e_{2}, \ldots \ldots \ldots \ldots, \varphi e_{n-1}, \xi\right\}$ is a also local orthonormal basis of vector fields on indefinite trans-Sasakian manifold $M^{n}$. Now, putting $X=U=e_{i}$ in equation (5.6) and taking summation over $i, 1 \leq i \leq n-1$, we have

$$
\begin{aligned}
& \sum_{i=1}^{n-1} g\left(R\left(\varphi e_{i}, Y\right) Z, \varphi e_{i}\right) \\
= & \frac{1}{(n-1)} \sum_{i=1}^{n-1}\left[S(Y, Z) g\left(\varphi e_{i}, \varphi e_{i}\right)-S\left(\varphi e_{i}, Z\right) g\left(Y, \varphi e_{i}\right)\right] \\
& -\frac{(\beta+\varepsilon)}{(n-1)} \sum_{i=1}^{n-1} g\left(\varphi e_{i}, Z\right) g\left(Y, \varphi e_{i}\right)+\frac{(\beta+\varepsilon)}{(n-1)} \sum_{i=1}^{n-1} g(Y, Z) g\left(\varphi e_{i}, \varphi e_{i}\right) \\
& -\frac{(1+\varepsilon \beta)}{(n-1)} \sum_{i=1}^{n-1} \eta(Y) \eta(Z) g\left(\varphi e_{i}, \varphi e_{i}\right)+\frac{\varepsilon \alpha}{(n-1)} \sum_{i=1}^{n-1} \eta\left(e_{i}\right) \eta(Z) g\left(Y, \varphi e_{i}\right) \\
& -\frac{\alpha}{(n-1)} \sum_{i=1}^{n-1} g\left(e_{i}, Z\right) g\left(Y, \varphi e_{i}\right)-\frac{\alpha}{(n-1)} \sum_{i=1}^{n-1} g(\varphi Y, Z) g\left(\varphi e_{i}, \varphi e_{i}\right) \\
+ & \alpha \sum_{i=1}^{n-1} g(Y, Z) g\left(e_{i}, \varphi e_{i}\right)+\alpha \sum_{i=1}^{n-1} g\left(\varphi e_{i}, Z\right) g\left(\varphi Y, \varphi e_{i}\right) .
\end{aligned}
$$

We have also

$$
\sum_{i=1}^{n-1} S\left(\varphi e_{i}, Z\right) g\left(Y, \varphi e_{i}\right)=S(Y, Z)-\varepsilon g(\xi, Z) S(Y, \xi) .
$$

Now, using equations $(2.1),(2.2),(2.4),(2.16),(4.8)-(4.16)$ and (5.8) in equation (5.7), we have

$$
\begin{aligned}
S(Y, Z)= & {\left[(n-2)(\varepsilon+\beta)+\varepsilon(n-1)\left(\alpha^{2}-\beta^{2}\right)-(n-1)(\xi \beta)\right] g(Y, Z) } \\
& +[\varepsilon(n-2)(\xi \beta)-(n-2)(1+\varepsilon \beta)] \eta(Y) \eta(Z) \\
& -[2 \varepsilon(n-1) \alpha \beta+(n-1)(\xi \alpha)-\alpha] g(\varphi Y, Z) \\
& -\varepsilon \eta(Y)(\varphi Z) \alpha-\varepsilon(n-2)(\xi \beta) \eta(Y) .
\end{aligned}
$$

If $\alpha=0$ and $\beta=$ constant in equation (5.9), we get

$$
S(Y, Z)=\left[(n-2)(\varepsilon+\beta)-(n-1) \varepsilon \beta^{2}\right] g(Y, Z)+(2-n)(1+\varepsilon \beta) \eta(Y) \eta(Z) .
$$

Therefore,

$$
S(Y, Z)=a g(Y, Z)+b \eta(Y) \eta(Z),
$$

where $a=(n-2)(\varepsilon+\beta)-(n-1) \varepsilon \beta^{2}$ and $b=(2-n)(1+\varepsilon \beta)$.

This result shows that the manifold under the consideration is an $\eta$ - Einstein manifold. Thus, we can state the following theorem:

Theorem 5.2. An $n$-dimensional quasi projectively flat indefinite trans-Sasakin manifold $M^{n}$ with respect to a semi-symmetric metric connection is $\eta$-Einstein manifold if $\alpha=0$ and $\beta=$ constant. 


\section{$6 \varphi$-Projectively flat indefinite trans Sasakian manifold with respect to a semi-symmetric metric connection}

An $M^{n}$ indefinite trans-Sasakian manifold with respect to a semi-symmetric metric connection is said be $\varphi$-projectively flat if

$$
\varphi^{2}(\bar{P}(\varphi X, \varphi Y) \varphi Z)=0
$$

where $\bar{P}$ is the projective curvature tensor of the $M^{n}$ indefinite trans-Sasakian manifold with respect to a semi-symmetric metric connection. Suppose $M^{n}$ be a $\varphi$-projectively flat indefinite trans-Sasakian manifold with respect to a semi-symmetric metric connection . It is known that $\varphi^{2}(\bar{P}(\varphi X, \varphi Y) \varphi Z)=0$ holds if and only if

$$
g(\bar{P}(\varphi X, \varphi Y) \varphi Z), \varphi U)=0
$$

for any $X, Y, Z, U \in T M^{n}$. Replace $Y=\varphi Y$ and $U=\varphi U$ in the equation (5.4), we have

$$
\begin{aligned}
g(\bar{P}(\varphi X, Y \varphi) \varphi Z, \varphi U)= & g(\bar{R}(\varphi X, \varphi Y) \varphi Z, \varphi U)-\frac{1}{(n-1)} \\
& {[\bar{S}(\varphi Y, \varphi Z) g(\varphi X, \varphi U)-\bar{S}(\varphi X, \varphi Z) g(\varphi Y, \varphi U)] . }
\end{aligned}
$$

From equations (6.2) and (6.3), we have

$$
\begin{aligned}
g(\bar{R}(\varphi X, \varphi Y) \varphi Z, \varphi U)= & \frac{1}{(n-1)}[\bar{S}(\varphi Y \varphi, Z) g(\varphi X, \varphi U) \\
& -\bar{S}(\varphi X, \varphi Z) g(\varphi Y, \varphi U)] .
\end{aligned}
$$

Using equations $(2.1),(2.2),(2.4),(2.5),(4.2)$ and (4.17) in equation (6.4), we have

$$
\begin{aligned}
& g(R(\varphi X, \varphi Y) \varphi Z, \varphi U) \\
= & \frac{1}{(n-1)}[S(\varphi Y, \varphi Z) g(\varphi X, \varphi U)-S(\varphi X, \varphi Z) g(\varphi Y, \varphi U)] \\
& +\frac{(\varepsilon+\beta)}{(n-1)} g(\varphi Y, \varphi Z) g(\varphi X, \varphi U)-\frac{(\varepsilon+\beta)}{(n-1)} g(\varphi X, \varphi Z) g(\varphi Y, \varphi U) \\
& +\frac{\alpha}{(n-1)} g(Y, \varphi Z) g(\varphi X, \varphi U)-\frac{\alpha}{(n-1)} g(X, \varphi Z) g(\varphi Y, \varphi U) \\
& +\alpha g(\varphi Y, \varphi Z) g(X, \varphi U)-\alpha g(\varphi X, \varphi Z) g(Y, \varphi U) .
\end{aligned}
$$

Let $\left\{e_{1}, e_{2}, \ldots \ldots \ldots \ldots, e_{-1}, \xi\right\}$ be a local orthonormal basis of vector fields on indefinite trans-Sasakian manifold $M^{n}$, then $\left\{\varphi e_{1}, \varphi e_{2}, \ldots \ldots \ldots \ldots, \varphi e_{n-1}, \xi\right\}$ is a also local orthonormal basis of vector fields on indefinite trans-Sasakian manifold $M^{n}$. Now, replace $X=U=e_{i}$ in equation (6.5) and taking 
summation over $i, 1 \leq i \leq n-1$, we get

$$
\begin{aligned}
& \sum_{i=1}^{n-1} g\left(R\left(\varphi e_{i}, \varphi Y\right) \varphi Z, \varphi e_{i}\right) \\
= & \frac{1}{(n-1)} \sum_{i=1}^{n-1}\left[S(\varphi Y, \varphi Z) g\left(\varphi e_{i}, \varphi e_{i}\right)-S\left(\varphi e_{i}, \varphi Z\right) g\left(\varphi Y, \varphi e_{i}\right)\right] \\
& +\frac{(\varepsilon+\beta)}{(n-1)} \sum_{i=1}^{n-1} g(\varphi Y, \varphi Z) g\left(\varphi e_{i}, \varphi e_{i}\right)-\frac{(\varepsilon+\beta)}{(n-1)} \sum_{i=1}^{n-1} g\left(\varphi e_{i}, \varphi Z\right) g\left(\varphi Y, \varphi e_{i}\right) \\
& +\frac{\alpha}{(n-1)} \sum_{i=1}^{n-1} g(Y, \varphi Z) g\left(\varphi e_{i}, \varphi e_{i}\right)-\frac{\alpha}{(n-1)} \sum_{i=1}^{n-1} g\left(e_{i}, \varphi Z\right) g\left(\varphi Y, \varphi e_{i}\right) \\
& +\alpha \sum_{i=1}^{n-1} g(\varphi Y, \varphi Z) g\left(e_{i}, \varphi e_{i}\right)-\alpha \sum_{i=1}^{n-1} g\left(\varphi e_{i}, \varphi Z\right) g\left(Y, \varphi e_{i}\right)
\end{aligned}
$$

Now, using equations $(2.1),(2.2),(2.4),(2.5),(2.16),(4.8)-(4.16)$ and $(5.8)$ in $(6.6)$, we have

$$
\begin{aligned}
& S(Y, Z) \\
= & {\left[(n-2)(\varepsilon+\beta)+\varepsilon(n-1)\left(\alpha^{2}-\beta^{2}\right)-(n-1)(\xi \beta)\right] g(Y, Z) } \\
& +[2 \varepsilon(n-2)(\xi \beta)-(n-2)(1+\varepsilon \beta)] \eta(Y) \eta(Z) \\
& +[\alpha-2 \varepsilon \alpha \beta(n-1)-(n-1)(\xi \alpha)] g(\varphi Y, Z) \\
& -[\varepsilon(\varphi Z) \alpha+\varepsilon(n-2)(Z \beta)] \eta(Y)-[\varepsilon(\varphi Y) \alpha+\varepsilon(n-2)(Y \beta)] \eta(Z),
\end{aligned}
$$

If $\alpha=0$ and $\beta=$ constant in equation (6.7), we get

$$
S(Y, Z)=\left[(n-2)(\varepsilon+\beta)-(n-1) \varepsilon \beta^{2}\right] g(Y, Z)+(2-n)(1+\varepsilon \beta) \eta(Y) \eta(Z) .
$$

Therefore

$$
S(Y, Z)=a g(Y, Z)+b \eta(Y) \eta(Z),
$$

where $a=(n-2)(\varepsilon+\beta)-(n-1) \varepsilon \beta^{2}$ and $b=(2-n)(1+\varepsilon \beta)$.

This result shows that the manifold under the consideration is an $\eta$ - Einstein manifold. Thus, we can state the following theorem:

Theorem 6.1. An $n$-dimensional $\varphi$-projectively flat indefinite trans-Sasakin manifold $M^{n}$ with semi-symmetric metric connection is $\eta$-Einstein manifold if $\alpha=0$ and $\beta=$ constant.

\section{Indefinite trans-Sasakian manifold with a semi-symmetric metric connection satisfying $\bar{R} \cdot \bar{S}=0$}

Now, suppose that an $M^{n}$ be $n$-dimensional indefinite trans-Sasakian manifold with a semisymmetric metric connection $\bar{\nabla}$ satisfying the condition

$$
\bar{R}(X, Y) \cdot \bar{S}=0 \text {. }
$$


Then, we have

$$
\bar{S}(\bar{R}(X, Y) Z, U)+\bar{S}(Z, \bar{R}(X, Y) U)=0
$$

Now, replace $X=\xi$ in equation (7.2), using equations (2.11) and (4.2), we have

$$
\begin{aligned}
& \varepsilon\left(\alpha^{2}-\beta^{2}\right) g(Y, Z) \bar{S}(\xi, U)-\left(\alpha^{2}-\beta^{2}\right) \eta(Z) \bar{S}(Y, U)-2 \varepsilon \alpha \beta g(\varphi Y, Z) \bar{S}(\xi, U) \\
& +2 \alpha \beta \eta(Z) \bar{S}(\varphi Y, U)+\varepsilon(Z \alpha) \bar{S}(\varphi Y, U)-\varepsilon g(\varphi Y, Z) \bar{S}(\operatorname{gard} \alpha, U) \\
& -\varepsilon g(\varphi Y, \varphi Z) \bar{S}(\operatorname{gard} \beta, U)+\varepsilon(Z \beta) \bar{S}(Y, U)-\varepsilon(Z \beta) \eta(Y) \bar{S}(\xi, U) \\
& -\beta g(Y, Z) \bar{S}(\xi, U)+\varepsilon \beta \eta(Z) \bar{S}(Y, U)+\alpha g(\varphi Y, Z) \bar{S}(\xi, U)-\varepsilon \alpha \eta(Z) \bar{S}(\varphi Y, U) \\
& +\varepsilon\left(\alpha^{2}-\beta^{2}\right) g(Y, U) \bar{S}(\xi, Z)-\left(\alpha^{2}-\beta^{2}\right) \eta(U) \bar{S}(Y, Z)-2 \varepsilon \alpha \beta g(\varphi Y, U) \bar{S}(\xi, Z) \\
& +2 \alpha \beta \eta(U) \bar{S}(\varphi Y, Z)+\varepsilon(U \alpha) \bar{S}(\varphi Y, Z)-\varepsilon g(\varphi Y, U) \bar{S}(\operatorname{gard}, Z) \\
& -\varepsilon g(\varphi Y, \varphi U) \bar{S}(\operatorname{gard} \beta, Z)+\varepsilon(U \beta) \bar{S}(Y, Z)-\varepsilon(U \beta) \eta(Y) \bar{S}(\xi, Z) \\
& -\beta g(Y, U) \bar{S}(\xi, Z)+\varepsilon \beta \eta(U) \bar{S}(Y, Z)+\alpha g(\varphi Y, U) \bar{S}(\xi, Z)-\varepsilon \alpha \eta(U) \bar{S}(\varphi Y, Z) \\
= & 0 .
\end{aligned}
$$

Using equations $(2.1)-(2.5),(2.12),(2.13),(4.17)$ and $(4.21)-(4.26)$ in equation (7.3), we get

$$
\begin{aligned}
& {\left[\left(\alpha^{2}-\beta^{2}\right)-\varepsilon(\xi \beta)-\varepsilon \beta\right] S(Y, Z) } \\
= & {\left[\varepsilon(n-1)\left(\alpha^{2}-\beta^{2}\right)^{2}-2 \beta(n-1)\left(\alpha^{2}-\beta^{2}\right)-2(n-1)\left(\alpha^{2}-\beta^{2}\right)(\xi \beta)\right.} \\
& +2 \varepsilon \beta(n-1)(\xi \beta)+\varepsilon(\xi \beta)^{2}+(\varphi g a r d \beta) \alpha+(n-2)(\operatorname{grad} \beta)^{2} \\
& +\varepsilon \beta^{2}(n-2)+(2 \beta+\varepsilon)(n-2)\left(\alpha^{2}-\beta^{2}\right)+\beta\left(\alpha^{2}-\beta^{2}\right) \\
& -2 \alpha^{2} \beta(n-2)-\varepsilon \alpha(\xi \alpha)-(2 \varepsilon \beta+1)(n-2)(\xi \beta)-\varepsilon \beta(\xi \beta) \\
& \left.-\varepsilon \beta(2 \beta+\varepsilon)(n-2)+\varepsilon \alpha^{2}(n-2)\right] g(Y, Z)+[-\varepsilon(\varphi g a r d \beta) \alpha \\
& -\varepsilon(n-2)(\operatorname{grad} \beta)^{2}-(n-2)(1+\varepsilon \beta)\left(\alpha^{2}-\beta^{2}\right) \\
& +2 \varepsilon \alpha^{2} \beta(n-2)+\alpha(n-2)(\xi \alpha)+(\varepsilon+\beta)(n-2)(\xi \beta) \\
& \left.+\beta(\varepsilon+\beta)(n-2)-\alpha^{2}(n-2)\right] \eta(Y) \eta(Z)+\left[-2 \varepsilon \alpha \beta(n-1)\left(\alpha^{2}-\beta^{2}\right)\right. \\
& +2(n-2) \alpha \beta^{2}+2 \alpha \beta(n-1)(\xi \beta)-(n-1)\left(\alpha^{2}-\beta^{2}\right)(\xi \alpha) \\
& +\varepsilon \beta(n-2)(\xi \alpha)+\varepsilon(\xi \alpha)(\xi \beta)+(\varphi g r a d \alpha) \alpha+(n-2) \\
& g(g r a d \alpha, g r a d \beta)+\alpha\left(\alpha^{2}-\beta^{2}\right)-\varepsilon \alpha(\xi \beta)-2 \alpha \beta(n-2)(\varepsilon+2 \beta) \\
& -(1+2 \varepsilon \beta)(n-2)(\varepsilon \alpha)+\varepsilon \alpha(\varepsilon+2 \beta)(n-2)] g(\varphi Y, Z) \\
& +[\varepsilon(\xi \alpha)+2 \alpha \beta-\varepsilon \alpha] S(\varphi Y, Z)+[(n-2)(\xi \beta)(Z \beta) \\
& -\varepsilon\left(\alpha^{2}-\beta^{2}\right)(\varphi Z) \alpha-\varepsilon(n-2)\left(\alpha^{2}-\beta^{2}\right)(Z \beta)+(\xi \beta)(\varphi Z) \alpha \\
& +\beta(\varphi Z) \alpha+\beta(n-2)(Z \beta)] \eta(Y)+\left[\varepsilon\left(\alpha^{2}-\beta^{2}\right)(\varphi Y) \alpha\right. \\
& +\varepsilon(n-2)\left(\alpha^{2}-\beta^{2}\right)(Y \beta)-2 \varepsilon \alpha \beta\left(\varphi^{2} Y\right) \alpha-2 \varepsilon \alpha \beta(n-2)(\varphi Y \beta) \\
& \left.-\beta(\varphi Y) \alpha-\beta(n-2)(Y \beta)+\alpha\left(\varphi^{2} Y\right) \alpha+\alpha(n-2)(\varphi Y \beta)\right] \eta(Z) \\
& -(Z \alpha)\left(\varphi^{2} Y\right) \alpha-(n-2)(Z \alpha)(\varphi Y \beta)-(Z \beta)(\varphi Y) \alpha \\
& -(n-2)(Y \beta)(Z \beta)+(n-2)(Z \beta)(\xi \beta) .
\end{aligned}
$$


If $\alpha=0$ and $\beta=$ constant in equation (6.7), we get

$$
S(Y, Z)=a g(Y, Z)+b \eta(Y) \eta(Z),
$$

where $a=-\left[\frac{(n-1) \varepsilon \beta^{4}+(n-2)(\operatorname{gard} \beta)^{2}+(n-2) \varepsilon \beta^{2}-(n-2)(\varepsilon+2 \beta) \beta^{2}-(n-2) \varepsilon \beta(\varepsilon+2 \beta)+(2 n-3) \beta^{3}}{\beta(\varepsilon+\beta)}\right]$

and $b=-\left[\frac{(n-2)(1+\varepsilon \beta) \beta^{2}+(n-2)(\varepsilon+\beta) \beta-(n-2) \varepsilon(\operatorname{grad} \beta)^{2}}{\beta(\varepsilon+\beta)}\right]$.

This shows that $M^{n}$ is an $\eta$-Einstein manifold. Thus, we can state the following:

Theorem 7.1. If an indefinite trans-Sasakian manifold with a semi symmetric metric connection $\bar{\nabla}$ satisfies $\bar{R} \cdot \bar{S}=0$, then the indefinite trans-Sasakian manifold is an $\eta$ - Einstein manifold if $\alpha=0$ and $\beta=$ constant.

\section{Indefinite trans-Sasakian manifold with respect to a semi-symmetric metric connection satisfies $\bar{P} \cdot \bar{S}=0$}

Now, we consider an indefinite trans-Sasakian manifold with a semi-symmetric connection $\bar{\nabla}$ satisfying

$$
(\bar{P}(X, Y) \cdot \bar{S})(Z, U)=0
$$

where $\bar{P}$ is the projective curvature tensor and $\bar{S}$ is the Ricci tensor with a semi-symmetric metric connection. Then, we have

$$
\bar{S}(\bar{P}(X, Y) Z, U)+\bar{S}(Z, \bar{P}(X, Y) U)=0
$$

Replace $X=\xi$ in the equation (8.2), we get

$$
\bar{S}(\bar{P}(\xi, Y) Z, U)+\bar{S}(Z, \bar{P}(\xi, Y) U)=0
$$

Putting $X=\xi$ in equation (5.1), we get

$$
\bar{P}(\xi, Y) Z=\bar{R}(\xi, Y) Z-\frac{1}{(n-1)}[\bar{S}(Y, Z) \xi-\bar{S}(\xi, Z) Y
$$

Using equations $(2.1),(2.2),(2.4),(2.11),(4.2),(4.17),(4.23)$ and (8.4) in equation (8.3), we get 


$$
\begin{aligned}
& \quad \frac{\varepsilon(n-1)\left(\alpha^{2}-\beta^{2}\right)+(n-2)(\varepsilon+\beta)}{(n-1)} g(Y, Z) \bar{S}(\xi, U)-\frac{1}{(n-1)} S(Y, Z) \bar{S}(\xi, U) \\
& -\frac{(n-2)}{(n-1)}(1+\varepsilon \beta) \eta(Y) \eta(Z) \bar{S}(\xi, U)+\frac{\alpha-2(n-1) \varepsilon \alpha \beta}{(n-1)} g(\varphi Y, Z) \bar{S}(\xi, U) \\
& -\varepsilon g(\varphi Y, Z) \bar{S}(g r a d \alpha, U)-\varepsilon g(\varphi Y, \varphi Z) \bar{S}(\operatorname{grad} \beta, U)+2 \alpha \beta \eta(Z) \bar{S}(\varphi Y, U) \\
& +\varepsilon(Z \alpha) \bar{S}(\varphi Y, U)+\varepsilon(Z \beta) \bar{S}(Y, U)-\varepsilon(Z \beta) \eta(Y) \bar{S}(\xi, U)-\varepsilon \alpha \eta(Z) \bar{S}(\varphi Y, U) \\
& -\frac{1}{(n-1)} \varepsilon(\xi \beta) \eta(Z) \bar{S}(Y, U)-\frac{(n-2)}{(n-1)} \varepsilon(Z \beta) \bar{S}(Y, U)-\frac{1}{(n-1)} \varepsilon(\varphi Z) \alpha \bar{S}(Y, U) \\
& +\frac{\varepsilon(n-1)\left(\alpha^{2}-\beta^{2}\right)+(n-2)(\varepsilon+\beta)}{(n-1)} g(Y, U) \bar{S}(\xi, Z)-\frac{1}{(n-1)} S(Y, U) \bar{S}(\xi, Z) \\
& -\frac{(n-2)}{(n-1)}(1+\varepsilon \beta) \eta(Y) \eta(U) \bar{S}(\xi, Z)+\frac{\alpha-2(n-1) \varepsilon \alpha \beta}{(n-1)} g(\varphi Y, U) \bar{S}(\xi, Z) \\
& -\varepsilon g(\varphi Y, U) \bar{S}(g r a d \alpha, Z)-\varepsilon g(\varphi Y, \varphi U) \bar{S}(g r a d \beta, Z)+2 \alpha \beta \eta(U) \bar{S}(\varphi Y, Z) \\
& +\varepsilon(U \alpha) \bar{S}(\varphi Y, Z)+\varepsilon(U \beta) \bar{S}(Y, Z)-\varepsilon(U \beta) \eta(Y) \bar{S}(\xi, Z)-\varepsilon \alpha \eta(U) \bar{S}(\varphi Y, Z) \\
& -\frac{1}{(n-1)} \varepsilon(\xi \beta) \eta(U) \bar{S}(Y, Z)-\frac{(n-2)}{(n-1)} \varepsilon(U \beta) \bar{S}(Y, Z)-\frac{1}{(n-1)} \varepsilon(\varphi U) \alpha \bar{S}(Y, Z) \\
& =\quad 0 .
\end{aligned}
$$

Putting $U=\xi$ and using equations (2.1) - (2.5), (4.17) and (4.21) - (4.26) in equation (8.5), we get

$$
\begin{aligned}
& {\left[\left(\alpha^{2}-\beta^{2}\right)-\varepsilon(\xi \beta)-\varepsilon \beta\right] S(Y, Z) } \\
= & {\left[\varepsilon(n-1)\left(\alpha^{2}-\beta^{2}\right)^{2}+(n-2)(\varepsilon+\beta)\left(\alpha^{2}-\beta^{2}\right)-\beta(n-1)\left(\alpha^{2}-\beta^{2}\right)\right.} \\
& -\beta(n-2)(1+\varepsilon \beta)-2(n-1)(\xi \beta)\left(\alpha^{2}-\beta^{2}\right)-(n-2)(1+\varepsilon \beta)(\xi \beta) \\
& -2 \alpha^{2} \beta(n-2)-\varepsilon \alpha(n-2)(\xi \alpha)+\varepsilon \alpha^{2}(n-2)+\varepsilon \beta(n-1)+\varepsilon(\xi \beta)^{2} \\
& \left.+(\varphi g r a d \alpha) \alpha+(n-2)(\operatorname{grad} \beta)^{2}\right] g(Y, Z)+\left[(n-2) \beta(\varepsilon+\beta)-(n-2)\left(\alpha^{2}-\beta^{2}\right)\right. \\
& +2(n-2) \varepsilon \alpha^{2} \beta+\alpha(n-2)(\xi \alpha)+(n-2)(\varepsilon+\beta)(\xi \beta)-\alpha^{2}(n-2) \\
& \left.-\varepsilon(\varphi g r a d \beta) \alpha-\varepsilon(n-2)(\operatorname{grad} \beta)^{2}\right] \eta(Y) \eta(Z)+\left[\alpha\left(\alpha^{2}-\beta^{2}\right)\right. \\
& -2(n-1) \varepsilon \alpha \beta\left(\alpha^{2}-\beta^{2}\right)-2 n \alpha \beta^{2}-\varepsilon \alpha(\xi \beta)-\varepsilon \beta(\xi \alpha)+2 \alpha \beta(\xi \beta) \\
& -2(n-2) \alpha \beta(2 \beta+\varepsilon)-(n-2)(1+2 \varepsilon \beta)(\xi \alpha)+\alpha(n-2)(2 \varepsilon \beta+1) \\
& -(n-1)\left(\alpha^{2}-\beta^{2}\right)(\xi \alpha)+(n-1) \varepsilon \beta(\xi \alpha)+\varepsilon(\xi \alpha)(\xi \beta)+(\varphi g r a d \alpha) \alpha \\
& +(n-2) g(g r a d \alpha, g r a d \beta)] g(\varphi Y, Z)+[2 \alpha \beta+\varepsilon(\xi \alpha)-\varepsilon \alpha] S(\varphi Y, Z) \\
& +\left[\varepsilon(n+3)\left(\alpha^{2}-\beta^{2}\right)(Z \beta)+\beta(n-2)(Z \beta)-\varepsilon\left(\alpha^{2}-\beta^{2}\right)(\varphi Z) \alpha\right. \\
& +(n-1) \beta(\varphi Z) \alpha+(\xi \beta)(\varphi Z) \alpha] \eta(Y)+\left[-2 \varepsilon \alpha \beta\left(\varphi^{2} Y\right) \alpha-2 \varepsilon \alpha \beta(n-2)(\varphi Y \beta)\right. \\
& +\alpha\left(\varphi^{2} Y\right) \alpha+\alpha(n-2)(\varphi Y \beta)+\varepsilon\left(\alpha^{2}-\beta^{2}\right)(\varphi Y) \alpha+\varepsilon(n-2)\left(\alpha^{2}-\beta^{2}\right)(Y \beta) \\
& -\beta(\varphi Y) \alpha-\beta(n-2)(Y \beta)] \eta(Z)-(Z \alpha)\left(\varphi^{2} Y\right) \alpha-(n-2)(Z \beta)(\varphi Y \beta) \\
& -(Z \beta)(\varphi Y) \alpha-\beta(n-2)(Y \beta) .
\end{aligned}
$$


If $\alpha=0$ and $\beta=$ constant in equation (8.6), we get

$$
S(Y, Z)=a g(Y, Z)+b \eta(Y) \eta(Z),
$$

where $a=-\left[\frac{(n-1) \varepsilon \beta^{4}-(n-2) \beta^{2}(\varepsilon+\beta)+(n-1) \beta^{3}-(n-2) \beta\left(1+\varepsilon \beta+(n-1) \varepsilon \beta+(n-2)(\operatorname{grad} \beta)^{2}\right.}{\beta(\varepsilon+\beta)}\right]$

and $b=-\left[\frac{(n-2) \beta(\varepsilon+\beta)+(n-2) \beta^{2}-(n-2) \varepsilon(\operatorname{grad} \beta)^{2}}{\beta(\varepsilon+\beta)}\right]$.

This result shows that the manifold under the consideration is an $\eta$-Einstein manifold. Thus,we can state the following theorem:

Theorem 8.1. If an indefinite trans-Sasakian manifold with a semi symmetric metric connection $\bar{\nabla}$ satisfies $\bar{P} \cdot \bar{S}=0$, then the indefinite trans-Sasakian manifold is an $\eta$ - Einstein manifold if $\alpha=0$ and $\beta=$ constant.

\section{Weyl conformal curvature tensor on indefinite trans-Sasakian manifold with a semi-symmetric metric connection}

The weyl conformal curvature tensor $\bar{C}$ of type $(1,3)$ of $M^{n}$ an $n$-dimensional indefinite transSasakian manifold with semi-symmtric metric connection $\bar{\nabla}$ is given by [16]

$$
\begin{aligned}
\bar{C}(X, Y) Z= & \bar{R}(X, Y) Z-\frac{1}{(n-2)}[\bar{S}(Y, Z) X-\bar{S}(X, Z) Y+g(Y, Z) \bar{Q} X \\
& -g(X, Z) \bar{Q} Y]+\frac{\bar{r}}{(n-1)(n-2)}[g(Y, Z) X-g(X, Z) Y],
\end{aligned}
$$

where $\bar{Q}$ is the Ricci operator with respect to the semi-symmetric metric connection $\bar{\nabla}$. Let $M^{n}$ be an $n$-dimensional indefinite trans-Sasakian manifold. The Weyl conformal curvature tensor $\bar{C}$ of $M^{n}$ with respect to the semi-symmetric metric connection $\bar{\nabla}$ is defined in equation (9.1).

Now, taking inner product with $U$ in equation (9.1), we get

$$
\begin{aligned}
& g(\bar{C}(X, Y) Z, U) \\
= & g(\bar{R}(X, Y) Z, U)-\frac{1}{(n-2)}[\bar{S}(Y, Z) g(X, U)-\bar{S}(X, Z) g(Y, U) \\
& +g(Y, Z) g(\bar{Q} X, U)-g(X, Z) g(\bar{Q} Y, U)]+\frac{\bar{r}}{(n-1)(n-2)} \\
& {[g(Y, Z) g(X, U)-g(X, Z) g(Y, U)] . }
\end{aligned}
$$

Using equations $(2.4),(4.2),(4.17),(4.18)$ and (4.20) in equation (9.2), we get

$$
\begin{aligned}
& \bar{C}(X, Y, Z, U) \\
= & g(R(X, Y) Z, U)-\frac{1}{(n-2)}[S(Y, Z) g(X, U)-S(X, Z) g(Y, U) \\
& +g(Y, Z) g(Q X, U)-g(X, Z) g(Q Y, U)]+\frac{r}{(n-1)(n-2)} \\
& {[g(Y, Z) g(X, U)-g(X, Z) g(Y, U)] }
\end{aligned}
$$


where $g(\bar{C}(X, Y) Z, U)=\bar{C}(X, Y, Z, U)$ and $g(R(X, Y) Z, U)=C(X, Y, Z, U)$ are Weyl curvature tensor with respect to semi-symmetric metric connection and metric connection respectively. we have

$$
\bar{C}(X, Y, Z, U)=C(X, Y, Z, U)
$$

where

$$
\begin{aligned}
& C(X, Y, Z, U) \\
= & g(R(X, Y) Z, U)-\frac{1}{(n-2)}[S(Y, Z) g(X, U)-S(X, Z) g(Y, Z) \\
& +g(Y, Z) g(Q X, U)-g(X, Z) g(Q Y, U)]+\frac{r}{(n-1)(n-2)} \\
& {[g(Y, Z) g(X, U)-g(X, Z) g(Y, U)] . }
\end{aligned}
$$

Theorem 9.1. The Weyl conformal curvature tensor of an indefinite trans-Sasakian manifold $M^{n}$ with respect to a metric connection is equal to the weyl conformal curvature of with respect to semi-symmetric metric connection.

\section{Indefinite trans-Sasakian manifold with weyl conformal flat conditions with a semi-symmetric metric connection}

Let us consider that the indefinite trans-Sasakian manifold $M^{n}$ with respect to the semi-symmetric metric connection is Weyl conformally flat, that is $\bar{C}=0$. Then from equation (9.1), we get

$$
\begin{aligned}
\bar{R}(X, Y) Z= & \frac{1}{(n-2)}[\bar{S}(Y, Z) X-\bar{S}(X, Z) Y+g(Y, Z) \bar{Q} X \\
& -g(X, Z) \bar{Q} Y]-\frac{\bar{r}}{(n-1)(n-2)}[g(Y, Z) X-g(X, Z) Y]
\end{aligned}
$$

Now, taking the inner product of equation (10.1) with $U$. Then, we get

$$
\begin{aligned}
g(\bar{R}(X, Y) Z, U)= & \frac{1}{(n-2)}[\bar{S}(Y, Z) g(X, U)-\bar{S}(X, Z) g(Y, U) \\
& +g(Y, Z) g(\bar{Q} X, U)-g(X, Z) g(\bar{Q} Y, U)] \\
& -\frac{\bar{r}}{(n-1)(n-2)}[g(Y, Z) g(X, U) \\
& -g(X, Z) g(Y, U)] .
\end{aligned}
$$

Using equations $(2.4),(4.2),(4.17),(4.18)$ and (4.20) in equation (10.2), we get

$$
\begin{aligned}
g(R(X, Y) Z, U)= & \frac{1}{(n-2)}[S(Y, Z) g(X, U)-S(X, Z) g(Y, U) \\
& +g(Y, Z) g(Q X, U)-g(X, Z) g(Q Y, U)] \\
& -\frac{r}{(n-1)(n-2)}[g(Y, Z) g(X, U) \\
& -g(X, Z) g(Y, U)] .
\end{aligned}
$$


Putting $X=U=\xi$ in equation (10.3) and using equations (2.2), (2.3) and (2.4), we get

$$
\begin{aligned}
& g(R(\xi, Y) Z, \xi) \\
= & \frac{1}{(n-2)}[\varepsilon S(Y, Z)-\varepsilon \eta(Y) S(\xi, Z)+g(Y, Z) S(\xi, \xi) \\
& -\varepsilon \eta(Z) S(Y, \xi)]-\frac{r}{(n-1)(n-2)}[\varepsilon g(Y, Z)-\eta(Y) \eta(Z)],
\end{aligned}
$$

Where $g(Q Y, Z)=S(Y, Z)$.

Now, using equations $(2.12),(2.13)$ and $(2.16)$, we get

$$
\begin{aligned}
S(Y, Z)= & {\left[(\xi \beta)-\varepsilon\left(\alpha^{2}-\beta^{2}\right)+\frac{r}{(n-1)}\right] g(Y, Z)+[\varepsilon(n-4)(\xi \beta)} \\
& \left.+n\left(\alpha^{2}-\beta^{2}\right)-\frac{\varepsilon r}{(n-1)}\right] \eta(Y) \eta(Z)-[2 \varepsilon(n-2) \alpha \beta \\
& +(n-2)(\xi \alpha)] g(\varphi Y, Z)-[\varepsilon(\varphi Z) \alpha+\varepsilon(Z \beta)(n-2)] \eta(Y) \\
& -[\varepsilon(\varphi Y) \alpha+\varepsilon(n-2)(Y \beta)] \eta(Z) .
\end{aligned}
$$

If $\alpha=0$ and $\beta=$ constant in equation (8.6), we get

$$
S(Y, Z)=\left[\varepsilon \beta^{2}+\frac{r}{(n-1)}\right] g(Y, Z)+\left[-n \beta^{2}-\frac{\varepsilon r}{(n-1)}\right] \eta(Y) \eta(Z) .
$$

Therefore

$$
S(Y, Z)=a g(Y, Z)+b \eta(Y) \eta(Z)
$$

where $a=\left[\varepsilon \beta^{2}+\frac{r}{(n-1)}\right]$ and $b=\left[-n \beta^{2}-\frac{\varepsilon r}{(n-1)}\right]$.

This shows that $M^{n}$ is an $\eta$-Einstein manifold. Thus, we can state as follows:

Theorem 10.1. Let $M^{n}$ be a $n$-dimensional Weyl conformally flat indefinite trans-Sasakian manifold with respect to semi symmetric metric connection $\bar{\nabla}$ is an $\eta$-Einstein manifold if $\alpha=0$ and $\beta=$ constant .

Now, taking equation (9.1), we have

$$
\begin{aligned}
\bar{C}(X, Y) Z= & \bar{R}(X, Y) Z-\frac{1}{(n-2)}[\bar{S}(Y, Z) X-\bar{S}(X, Z) Y \\
& +g(Y, Z) \bar{Q} X-g(X, Z) \bar{Q} Y] \\
& +\frac{\bar{r}}{(n-1)(n-2)}[g(Y, Z) X-g(X, Z) Y] .
\end{aligned}
$$


Using equations (2.15), (4.2)(4.17), (4.18) and (4.20) in equation (10.7), we get

$$
\begin{aligned}
& \bar{C}(X, Y) Z \\
= & C(X, Y) Z+(2 \beta+\varepsilon)[g(X, Z) Y-g(Y, Z) X] \\
& +(\varepsilon+\beta)[\eta(X) g(Y, Z)-\eta(Y) g(X, Z)] \xi \\
& +(1+\varepsilon \beta) \eta(Z)[\eta(Y) X-\eta(X) Y]-\alpha[g(\varphi X, Z) Y \\
& -g(\varphi Y, Z) X-g(Y, Z) \varphi X+g(X, Z) \varphi Y]-\frac{1}{(n-2)} \\
& {[(1+\varepsilon \beta)(n-2) \eta(Y) \eta(Z)-((2 \beta+\varepsilon)(n-2)+\beta) g(Y, Z) X} \\
& +\alpha(n-2) g(\varphi Y, Z) X+((2 \beta+\varepsilon)(n-2)+\beta) g(X, Z) Y \\
& -(1+\varepsilon \beta)(n-2) \eta(X) \eta(Z) Y-\alpha(n-2) g(\varphi X, Z) Y \\
& -((2 \beta+\varepsilon)(n-2)+\beta) g(Y, Z) X+(\varepsilon+\beta)(n-2) g(Y, Z) \eta(X) \xi \\
& +\alpha(n-2) g(Y, Z) \varphi X+((2 \beta+\varepsilon)(n-2)+\beta) g(X, Z) Y \\
& -(\varepsilon+\beta)(n-2) g(X, Z) \eta(Y) \xi-\alpha(n-2) g(X, Z) \varphi Y] \\
& -\frac{2 \beta+(2 \beta+\varepsilon)(n-2)}{(n-2)}[g(Y, Z) X-g(X, Z) Y] .
\end{aligned}
$$

Let $X$ and $Y$ are orthogonal basis to $\xi$. Putting $Z=\xi$ and using equations (2.1), (2.2) and (2.4) in equation (10.8), we get

$$
\bar{C}(X, Y) \xi=C(X, Y) \xi .
$$

Theorem 10.2. A $n$-dimensional indefinite trans-Sasakian manifold $M^{n}$ is Weyl $\xi$-conformally flat with respect to the semi-symmetric metric connection if and only if the manifold is also weyl $\xi$-conformally flat with respect to the metric connection provided that the vector fields are horizontal vector fields.

Example 10.3. Let us consider a 3 -dimensional manifold $M=\left\{(x, y, z) \in R^{3}: z \neq 0\right\}$, where $(x, y, z)$ are the standard co-ordinates in $R^{3}$. we choose the vector fields

$$
e_{1}=-z\left(\frac{\partial}{\partial x}+y \frac{\partial}{\partial y}\right), e_{2}=-z \frac{\partial}{\partial y}, e_{3}=\frac{\partial}{\partial z}
$$

which are linearly independent at each point of $M$. Let $g$ be the Riemannian metric defined by

$$
\begin{aligned}
& g\left(e_{1}, e_{2}\right)=g\left(e_{2}, e_{3}\right)=g\left(e_{3}, e_{1}\right)=0, \\
& g\left(e_{1}, e_{1}\right)=g\left(e_{2}, e_{2}\right)=g\left(e_{3}, e_{3}\right)=\varepsilon .
\end{aligned}
$$

Let $\eta$ be the 1 -form defined by $\eta(X)=\varepsilon g(X, \xi)$ for any $X \in \chi(M)$ and $\varphi$ be the $(1,1)$ tensor field defined by

$$
\varphi\left(e_{1}\right)=e_{2}, \varphi\left(e_{2}\right)=-e_{1}, \varphi\left(e_{3}\right)=0 .
$$

Then using the linearity of $\varphi$ and $g$, we have

$$
\begin{aligned}
\varphi^{2} X & =-X+\eta(X) e_{3}, \eta\left(e_{3}\right)=1, \\
g(\varphi X, \varphi Y) & =g(X, Y)-\varepsilon \eta(X) \eta(Y),
\end{aligned}
$$


where $e_{3}=\xi$, the structure $(\varphi, \xi, \eta, g)$ defines an indefinite Trans-Sasakian structure on $M$. Let $\nabla$ the Levi-Civita connection with metric $g$, then we have

$$
\left[e_{1}, e_{2}\right]=\varepsilon\left(-y e_{2}-z^{2} e_{3}\right),\left[e_{1}, e_{3}\right]=\frac{1}{z} \varepsilon e_{1},\left[e_{2}, e_{3}\right]=\frac{1}{z} \varepsilon e_{2} .
$$

The Riemannian connection $\nabla$ of the metric $g$ is given by

$$
\begin{aligned}
2 g\left(\nabla_{X} Y, Z\right)= & X g(Y, Z)+Y g(Z, X)-Z g(X, Y)+g([X, Y], Z) \\
& -g([Y, Z], X)+g([Z, X], Y),
\end{aligned}
$$

which known as Koszul's formula Taking $e_{3}=\xi$ and using Koszul's formula, we have

$$
\begin{aligned}
\nabla_{e_{1}} e_{1} & =-\frac{1}{z} \varepsilon e_{3}, \nabla_{e_{1}} e_{2}=-\frac{1}{2} \varepsilon z^{2} e_{3}, \nabla_{e_{1}} e_{3}=\frac{1}{z} \varepsilon e_{1}+\frac{1}{2} \varepsilon z^{2} e_{2} \\
\nabla_{e_{2}} e_{1} & =\frac{1}{2} \varepsilon z^{2} e_{3}+\varepsilon y e_{2}, \nabla_{e_{2}} e_{2}=\varepsilon\left(-y e_{1}-\frac{1}{z} e_{3}\right), \nabla_{e_{3}} e_{3}=0 \\
\nabla_{e_{2}} e_{3} & =\frac{1}{z} \varepsilon e_{2}-\frac{1}{2} \varepsilon z^{2} e_{1}, \nabla_{e_{3}} e_{1}=\frac{1}{2} \varepsilon z^{2} e_{2}, \nabla_{e_{3}} e_{2}=-\frac{1}{2} \varepsilon z^{2} e_{1}
\end{aligned}
$$

Therefore, the semi-symmetric metric connection on $M$ is given by

$$
\begin{aligned}
\bar{\nabla}_{e_{1}} e_{1} & =-\frac{1}{z} \varepsilon e_{3}-\varepsilon e_{3}, \bar{\nabla}_{e_{1}} e_{2}=-\frac{1}{2} \varepsilon z^{2} e_{3}, \bar{\nabla}_{e_{3}} e_{3}=0 \\
\bar{\nabla}_{e_{1}} e_{3} & =\frac{1}{z} \varepsilon e_{1}+\frac{1}{2} \varepsilon z^{2} e_{2}+\varepsilon e_{1}, \quad \bar{\nabla}_{e_{3}} e_{2}=-\frac{1}{2} \varepsilon z^{2} e_{1} \\
\bar{\nabla}_{e_{2}} e_{1} & =\frac{1}{2} \varepsilon z^{2} e_{3}+\varepsilon y e_{2}, \bar{\nabla}_{e_{2}} e_{2}=\varepsilon\left(-y e_{1}-\frac{1}{z} e_{3}-e_{3}\right), \\
\bar{\nabla}_{e_{2}} e_{3} & =\frac{1}{z} \varepsilon e_{2}-\frac{1}{2} \varepsilon z^{2} e_{1}+\varepsilon e_{2}, \quad \bar{\nabla}_{e_{3}} e_{1}=\frac{1}{2} \varepsilon z^{2} e_{2} .
\end{aligned}
$$

Now, for $\xi=e_{3}$, above results satisfy

$$
\bar{\nabla}_{X} \xi=-\varepsilon \alpha(\varphi X)+(1+\varepsilon \beta)(X-\eta(X) \xi),
$$

with $\alpha=-\frac{1}{2} z^{2}$ and $\beta=\frac{1}{z}$. Consequently $M(\varphi, \xi, \eta, g, \varepsilon)$ is a indefinite trans Sasakian manifold with semi-symmetric connection.

Acknowledgement. Sushil Kumar is thankful to UGC, New Dehli (India) for a fellowship.

\section{References}

[1] C. S. Bagewadi, On totally real submanifolds of a Kahlerian manifold admitting Semi symmetric metric F-connection. Indian. J. Pure. Appl. Math, 13, 528-536, (1982) .

[2] C. S. Bagewadi and N. B. Gatti, On irrotational quasi-conformal curvature tensor. Tensor.N.S., 64, 284-258, (2003). 
[3] C. S. Bagewadi and E. Girish Kumar, Note on Trans-Sasakian Manifolds. Tensor. N. S., 65, 80-88 (2004).

[4] A. Bejancu and K. L. Duggal, Real hypersurfaces of idefinite Kahler manifolds. Int. J. Math. Math. sci., 16, 545-556, (1993). .

[5] D. E. Blair, Contact manifolds in Riemannian geometry. Lecture note in Mathematics, 509, Springer-Verlag Berlin-New York, 1976.

[6] U. C. De. and A. Sarkar, On ( $\varepsilon)$-Kenmotsu manifolds. Hadronic journal, 32 , 231-242, (2009).

[7] U. C. De and Absos Ali Shaikh, K-contact and Sasakian manifolds with conservative quasiconformal curvature tensor. Bull. Cal. Math. Soc., 89, 349-354, (1997).

[8] A. Friedmann and J. A. Schouten, Uber die geometric der holbsymmetrischen Ubertragurgen. Math. Zeitschr. 21, 211-233, (1924).

[9] H. A. Hayden, Subspaces of space with torsion. Proc. Lond. Math. Soc. 34 , 27-50, (1932) .

[10] S. I. Hussain and A. Sharafuddin, Semi-symmetric metric connections in almost contact manifolds. Tensor, N. S.,30, 133-139, (1976).

[11] Amur Kumar and S. S. Pujar, On Submanifolds of a Riemannian manifold admitting a metric semi-symmetric connection. Tensor, N. S., 32, 35-38, (1978).

[12] R. Kumar, R. Rani and R. K. Nagaich, On sectional curvature of $(\varepsilon)$-Sasakian manifolds. Int. J. Math. Math. sci., (2007), ID. 93562.

[13] J. C. Marrero, The local structures of trans-Sasakian manifolds. Ann. Mat. Pura. Appl, (4), 162, 77-86, (1992).

[14] Halammanavar G. Nagaraja, Rangaswami C. Premalatha and Ganganna Somashekara, On an $(\varepsilon, \delta)$ trans-Sasakian structure. Proceedins of the Estonian Academy of Sciences, (1), 61, 20-28 (2012).

[15] J. A. Oubina, New classes of almost contact metric structures. Publicationes Mathematicae Debrecen ,vol.32, 187-193, (1985).

[16] M. M. Tripathi, Ricci solitons in contact metric manifolds. arXiv:0801.4222v1,[math.DG],28, (2008).

[17] K. Yano, On semi-symmetric metric connections. Revue Roumaine de Math. Pures et Appliques., 15 1579-1586, (1970) .

[18] X.Xufeng and C. Xiaoli, Two theorems on $(\varepsilon)$-Sasakian manifolds. Int. J. Math. Sci., 21249 -254, (1998). 